\title{
Optimization design and simulation of giant magnetostrictive precise flow valve
}

\author{
Bin $\mathrm{Xu}^{1,2}$, Cuiqun $\mathrm{He}^{1,2}$, Quanguo $\mathrm{Lu}^{1,2}$, Hongmin Wen ${ }^{1,2}$, Gang Tang ${ }^{1,2}$,Zhifang \\ $\mathrm{Zhu}^{1,2}$, Zhibiao $\mathrm{Li}^{1,2}$, Wu Yang ${ }^{1,2}$ \\ ${ }^{1}$ Jiangxi Province Key Laboratory of precision drive and control \\ ${ }^{2}$ School of Mechanical \& Electrical Engineering, Nanchang Institute of Technology, Nanchang, \\ China
}

Keywords: giant magnetostrictive matiarial (GMM), flow valve, hydraulic amplifier, simulation

\begin{abstract}
This paper introduces a precise flow valve applying giant magnetostrictive material(GMM). The dimension of driving coil was optimized by theory study. A hydraulic amplifier is used in the flow valve for the first time as we know, which can amplify the extension of the Terfenol-D into a large displacement. Though the finite element analysis, the GMM precise valve can well adjust the flow rate by control the input current.
\end{abstract}

\section{Introduction}

With increasing requirements about environmental protection, safety, health and energy saving, water hydraulic technology has attracted many attentions as a solution. Water servo control technique, an indispensable part of water hydraulic technology, has become an important research direction all over the world [1, 2].

Magnetostriction is the change in shape of materials under the influence of an external magnetic field. The magnetostrictive effect was first described in the 19th century (1842) by an Engilsh physicist James Joule[3]. In 1970s, Doctor Clark of NAVSWC of the United States find some material have big magnetriction coefficient at room temperature, named giantmagnetostrictive material(GMM), its deformation rate is 100 times bigger than traditional magnetostrictive material like Co and Ni, 10-14 times bigger than PZT, its response frequency is as high as $20 \mathrm{kHz}[4]$.

GMM flow valve generally use Terfenol-D rod, a typical GMM, as its driving element, magnetic field is generated by the coil around the outside of the GMM rod, the magnetriction coefficient of the material increases with the increase of magnetic field intensity in a certain range. In order to improve the displacement output performance and energy conversion efficiency of the giant magnetostrictive valve, this paper optimize the design of the valve actuator. As the deformation rate of Terfenol-D rod is not large enough to drive the valve plug move in the range needed, we design a displacement amplification mechanism, it amplifies the deformation of the Terfonel-D rod at a certain ratio, and change the deformation into the displacement of the valve plug. Secondly, we discuss the optimization design of the coil of the actuator to get higher magnetic field intensity, so the actuator can output larger displacement.

\section{Design of GMM flow valve}

\section{Design of GMA for flow valve.}

As being restricted by the dimension of magnetic drive, its output displacement is small, in order to transfer large displacement, a amplifying mechanism must be set in the valve. There are several displacement amplification mechanism, such as lever amplifier, hydraulic amplifier, triangle amplifier and elliptical amplifier, etc[5]. Lever amplifier is not suitable for the GMM valve for its large size. Dual chip amplifier. triangle amplifier and elliptical amplifier have compact structure, but their structure are complicated and the amplification factor is small. Hydraulic amplifier has simple structure and big displacement amplification factor, as show in Fig.1. The GMM rod drive the piston move forward with a displacement of S1,as the oil can not be compressed, the piston 2 will move with a displacement of S2. 
$\pi d^{2} S_{1} / 4=\pi D^{2} S_{2} / 4$

so, the displacement of piston $2 \mathrm{~S}_{2}=\mathrm{S}_{1} \mathrm{D}^{2} / \mathrm{d}^{2}$, if the diameter of piston 1 is bigger than the piston 2 , the piston 2 can acquire a huge displacement.

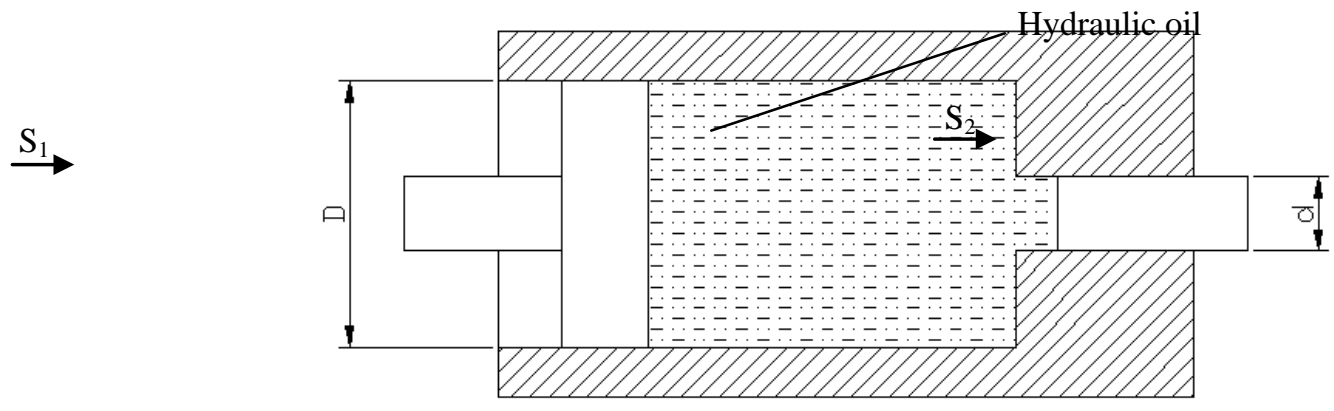

Fig. 1 the structure of hydraulic amplifier

The structure of GMM pump is show in Fig.2. The Terfenol-D rod is selected as the driven element. When DC current is input into the driven coil, the Terfenol-D rod will extend and push the big piston(part 8) move forward for S1. The hydraulic oil drives the small piston(part 6) move for $\mathrm{S} 2$. The constant $\mathrm{d}$ and $\mathrm{D}$ are the diameter of big piston and small piston respectively. As can be seen from Eq.1, S1:S2=D2:d2, so the spool(part 4) can achieve a huge amplification coefficient through the hydraulic displacement amplifier. In this paper, the diameter of big piston and small piston is $50 \mathrm{~mm}$ and $10 \mathrm{~mm}$ respectively, therefore the displacement amplification coefficient is 25 .

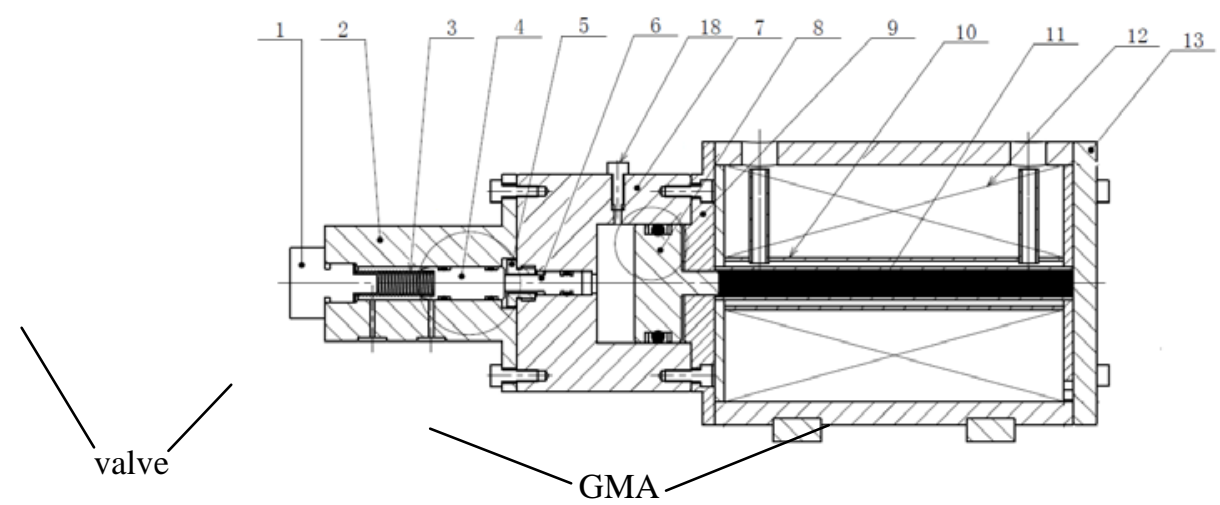

Fig. 2 the structure of GMM valve

The magnetostrictive coefficient $\lambda=\mathrm{d} l / l$, it means the length change of the Giant magnetostrictive material as a result of magnetic field change[6]. The relation of magnetostrictive coefficient to the strength of magnetic field is shown in Fig.3. The magnetostrictive coefficient increases with the strength driven magnetic filed till saturation. The direction of magnetic field will not increase the value of magetostrictive coefficient. And the relationship of magnetostrictive coefficient and the strength of magnetic field can be describe as follw:

$$
\lambda=\frac{3}{2} \frac{\lambda_{\mathrm{s}}}{M_{\mathrm{S}}{ }^{2}} M^{2}
$$

$\lambda_{\mathrm{s}}, M_{\mathrm{s}}$ and $M$ is the saturate magetostrictive coefficient, saturate magnetization and magnetization respectively. In order to increase the output displacement, the current of the coil should be enhanced, and the dimension of the driven coil and Terfenol-D rod should be well designed to reduce the resistance and improve the energy transition efficiency. 


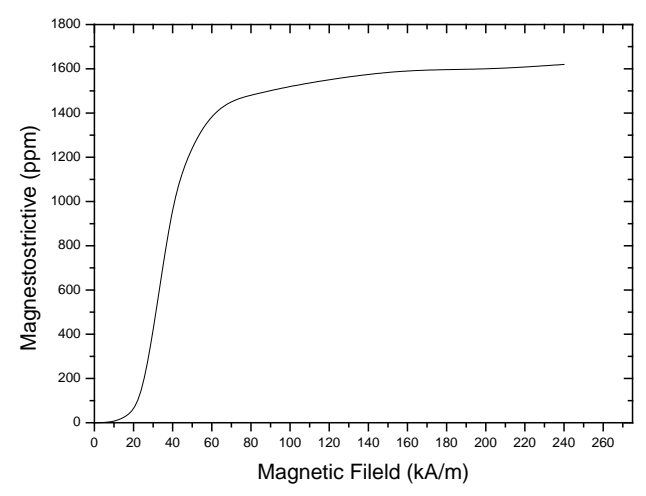

Fig.3 The relation of magnetostrictive coefficient versus magnetic field

\section{Design of driven coil}

In order to acquire big magnetic strength and improve the energy transition efficiency, the driven coil should be well designed, which concludes the dimension of the coil, the diameter of copper wire, the circles and so on. Since resistance and inductance of the coil will cause losses of the effective magnetic field of the magnetostrictive rod and reduce quality factor, so the design principle is reduce the coil resistance and inductance of the coil. First, the appropriate diameter need to be selected. The coil structure is shown in Figure 4, the coil length $L$ is known, the coil outer diameter $\mathrm{r} 2$ and inner diameter $\mathrm{r} 1$ can be within a certain range selection, $\mathrm{a} \leqslant \mathrm{r} 1<\mathrm{r} 2 \leqslant \mathrm{~b}$. Coil resistance and enameled wire diameter are related with and cross-sectional area, described as followed.

$$
R=\rho \frac{l}{S}=\rho \frac{N\left(r_{1}+r_{2}\right)}{\left(\frac{d}{2}\right)^{2}}=4 \rho \frac{L\left(r_{2}^{2}-r_{1}^{2}\right)}{d^{4}}
$$

Where $\rho$ is the resistivity of magnet wire, $l$ is the length of the enameled wire, $S$ is the crosssectional area enameled, $N$ is the total number of circles of the coil, $d$ is the diameter of the copper wire.

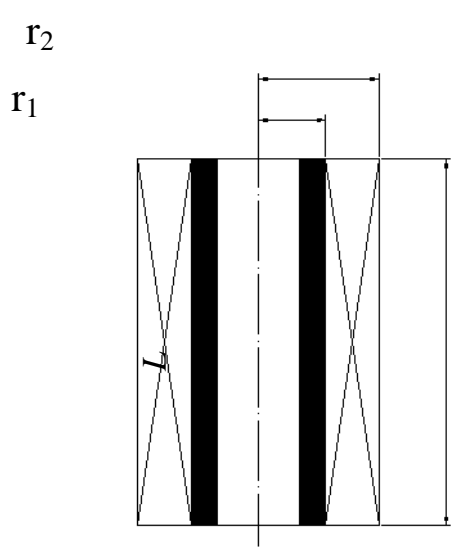

Fig.4 Schematic diagram of driving coil

Figure 5 shows the coil resistance under the different diameter and different inner diameter coil. The resistance is large and most of the energy supply is in the form of coil heat loss out while the diameter is $0.1 \mathrm{~mm}$ and the resistance decreases rapidly while the diameter of $0.2 \mathrm{~mm}$. While the diameter is greater than $0.3 \mathrm{~mm}$, the resistance remains stable. Inner diameter of the coil has little influence to the coil resistance within the within a predetermined range. Different coil wire with the same inner diameter have almost same resistance. Therefore, in the case of meeting the energy range, the diameter of the coil wire should above $0.2 \mathrm{~mm}$. Finally, According to Fig.5, coil of 2000 turns and $1.1 \mathrm{~mm}$ diameter should be chosen because the coil resistance is small and stable when $\mathrm{d}$ 
(diameter) is less than 1.1mm. However, as there is a magnetic leakage in GMM rod, a coil of 2055 turns is chosen finally. The magnetic induction at the central axis of coil can be obtained by BiotSavart law.

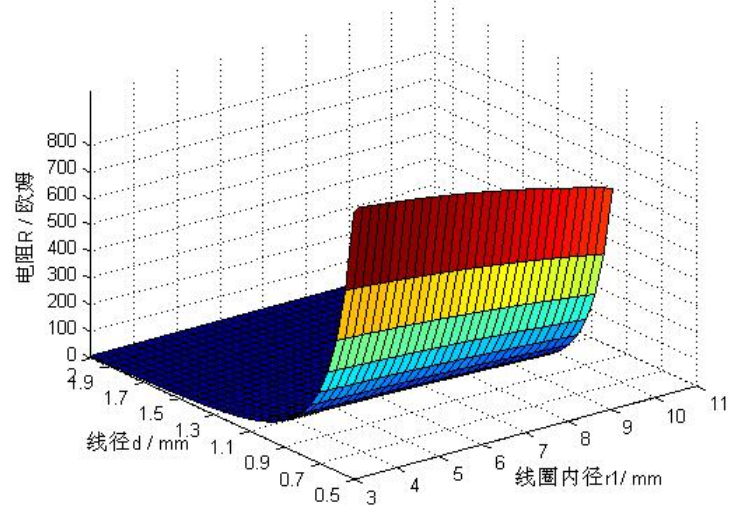

Fig.5 resistance versus thread diameter and inner diameter

$$
B=\mu \frac{N I}{2\left(r_{2}-r_{1}\right)} \ln \left(\frac{r_{2}+\sqrt{r_{2}^{2}+L^{2} / 4}}{r_{1}+\sqrt{r_{1}^{2}+L^{2} / 4}}\right)
$$

Where $\mu$ is magnetic permeability, $I$ is the coil current.

\section{Simulation}

\section{Simulation of the magnetic field}

Driving coil is undergoing a magnetic field simulation in Comsol and the results can be shown in Fig. 6 and Fig.7, which show a consistency between the simulation results and the theoretical analysis. The magnetic field intensity at the central axis is almost uniform.

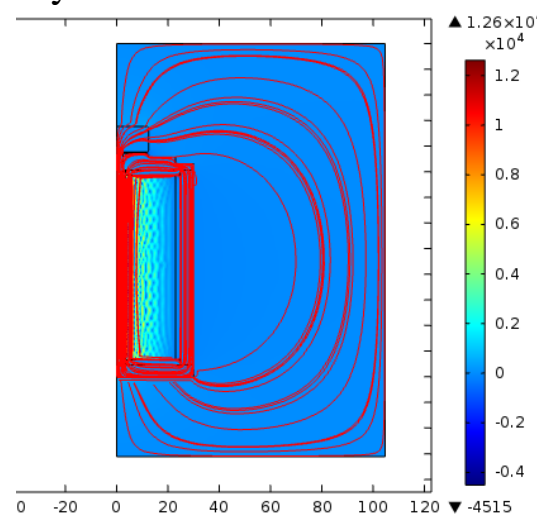

Fig.6 Driving coil magnetic flux diagram 


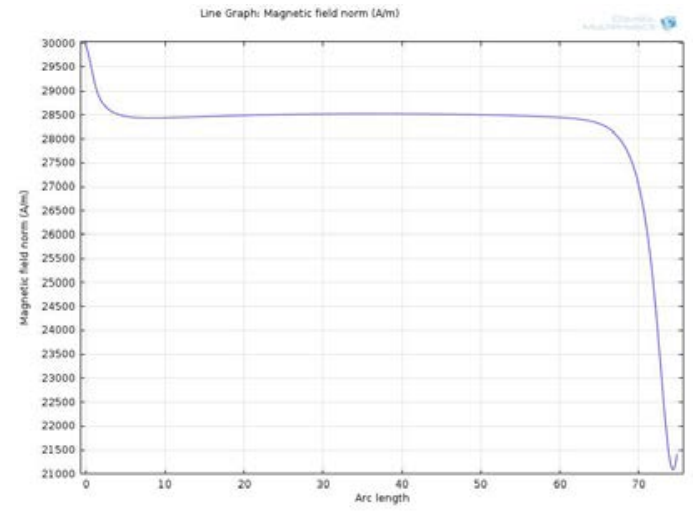

Fig.7 magnetic Field intensity at the central axis of coil

\section{Simulation of flow valve}

In order to simulate the flow velocity of the outlet respect to different valve opening( the value of $\mathrm{S}$ in Fig.8), the channel of valve is simplified as Fig.8. As a overflow valve was set in the hydraulic circuit, a overflow outlet was added in the simplified channel.

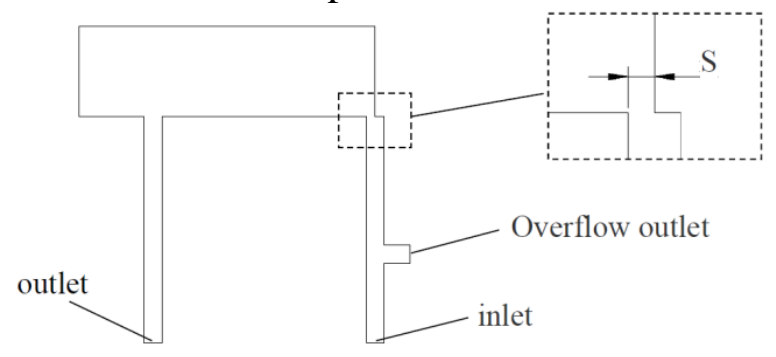

Fig.8 simplified channel of flow valve

The software Comsol is applied to simulate the fluid field of the valve channel. Hypothesis the entrance flow velocity is $1 \mathrm{~m} / \mathrm{s}$, flow field distribution in the valve channel is shown in Fig.9. The velocity of flow at outlet versus different valve opening is shown in Fig.10.

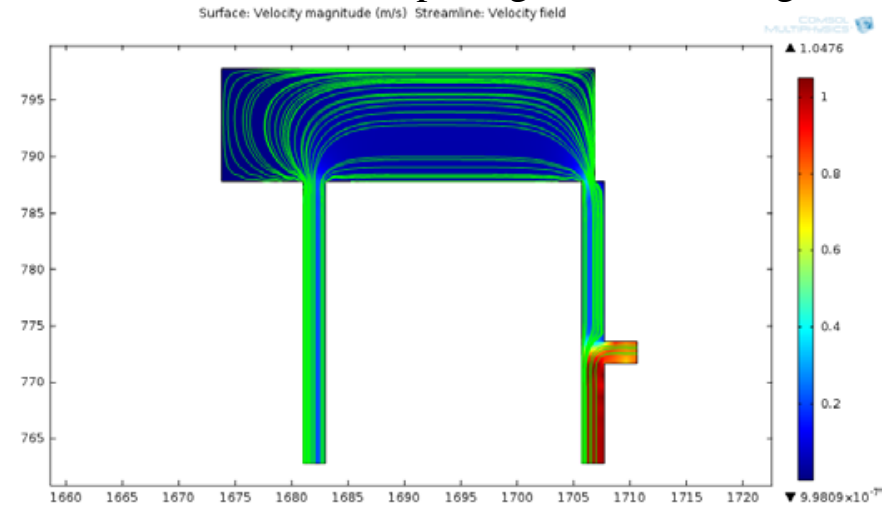

Fig.9 flow field distribution in the valve channel

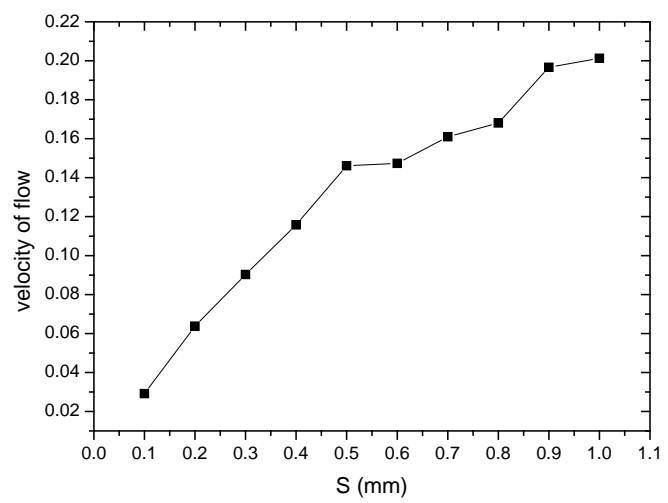

Fig.10 Velocity of flow in outlet versus different 


\section{Discussion}

As can be seen from the simulation, the The maximal magnetic field intensity at the central axis is $27 \mathrm{kA} / \mathrm{m}$. According to the curve of magnetostrictive coefficient versus magnetic field(Fig.3),the magnetostrictive coefficient can reach to $700 \mathrm{ppm}$, so the extension of Terfenol-D rod(140mm) is $98 \mu \mathrm{m}$. As the amplification coefficient of the hydraulic amplifier is 25 , the maximal displacement can reach to $2.45 \mathrm{~mm}$, which is larger than dimension of the valve opening, can fulfill the requirement of flow adjustment.

\section{Acknowledgments}

This work is supported by the financial support from the science and technology project (No.20122BBE500033) of Jiangxi provincial department of science and technology of China, the Science Fund of the Education Department of Jiangxi province of China (GJJ11628, GJJ13765), and the science and technology project(2012-KJZC-GY-JDYTH-003) of Nanchang bureau of science and technology of China .The authors are also grateful to colleagues for their essential contribution to this work.

\section{References}

[1] J.Yuh. Design and Control of Autonomous Underwater Robots: A Survey. Autonomous Robots Vol. 8(Jan. 2000), p.7-24. doi: 10.1023/A: 1008984701078.

[2] Wang Xinhua, Sun Shuwen, and Li Jianfeng. Present Situation \& Application Prospect of Hydraulic Pressure Servo-control Technological Development.Machine Tool and Hydraulic Pressure Vol. 36( May. 2008), p.177-181.

[3]A.Grunwald,A.G..Olabi*.Design of a magnetostrictive(MS) actuator.Sensors and Actuators A Vol.144(2008),p.161-175.

[4] CLARK A E. Ferromagnetic material[M]. Amsterdam:North2Holand Publishing Company(1980),p.531 588.

[5] Introduction of flexible chain. Harbin Institute of Technology Boshi Precision Measure\&Control Co.,Ltd. http://www.bsjm.com.cn/

[6] DAPINO M.J, SMITH R.C.,FLATAV A.B. An active and structural strain model for magtostrictive transdncers. SPIE conference on Smart Strutures and Integrated systerns , san Diego , 1998 , 3329 :198 209. 